\title{
Surgical Management of Paediatric Aphakia in the Absence of Sufficient Capsular Support
}

\author{
Evdoxia-Maria Karasavvidou $\mathbb{D}^{1},{ }^{1}$ Craig Wilde, ${ }^{2}$ Anwar Zaman, ${ }^{2}$ Gavin Orr, ${ }^{2}$ \\ Dharmalingam Kumudhan, ${ }^{2}$ and Georgios D. Panos $\mathbb{D}^{2}$ \\ ${ }^{1}$ Karasavvidou Eye Centre, Naousa, Greece \\ ${ }^{2}$ Department of Ophthalmology, Queen's Medical Centre, Nottingham University Hospitals NHS Trust, Nottingham, UK
}

Correspondence should be addressed to Georgios D. Panos; gdpanos@gmail.com

Received 4 November 2021; Accepted 15 November 2021; Published 4 December 2021

Academic Editor: Alessandro Meduri

Copyright (c) 2021 Evdoxia-Maria Karasavvidou et al. This is an open access article distributed under the Creative Commons Attribution License, which permits unrestricted use, distribution, and reproduction in any medium, provided the original work is properly cited.

\begin{abstract}
There are several available options for the demanding surgical correction of paediatric aphakia without sufficient capsular support. The literature suggests the implantation of a transscleral fixated posterior chamber-intraocular lens (PCIOL), an intrascleral fixated PCIOL, an iris-sutured intraocular lens (IOL), or an anterior chamber iris-claw IOL. We searched for reports on the management of paediatric aphakia in case of inadequate capsular support that delineated the diverse surgical approaches and their postoperative results. Analysis demonstrated that different complications can be encountered depending on IOL placement technique, such as suture rupture, IOL dislocation, secondary glaucoma, endophthalmitis, vitreous hemorrhage, and endothelial cell loss. However, it was shown that various IOL designs have similar visual outcomes. Taking into consideration the advantages and disadvantages of each surgical technique, ophthalmic surgeons can determine the safest and most efficient approach for paediatric aphakic patients.
\end{abstract}

\section{Introduction}

Paediatric aphakia with the absence of adequate capsular support may occur after lens removal for congenital cataract, after trauma or lens subluxation associated with systemic disorders. Refractive error can be temporarily managed with spectacles or contact lenses; however, secondary implantation of an intraocular lens (IOL) provides better visual outcomes in children with aphakia or lens subluxation $[1,2]$.

Various alternatives have been proposed for IOL secondary implantation through the years, but the research for the optimal surgical approach and IOL design is still in progress. To date, available options include the use of an anterior chamber IOL, a scleral fixated (SF) sutured posterior chamber IOL (PCIOL), an intrascleral fixated PCIOL, an iris-sutured PCIOL, or iris-claw lens [3]. Glued sutureless intrascleral fixation techniques as well as the creation of scleral pockets for intrascleral fixation of IOL haptics have shown good results in adults $[4,5]$. On the other hand, the implantation of anterior chamber IOLs has been related to several postoperative complications, such as corneal endothelial cell loss (ECL), glaucoma, intraocular inflammation, hyphema, and cystoid macular oedema. Thus, anterior chamber IOLs are not recommended for use in the paediatric population [6].

This review aims to present the different surgical methods used in cases of paediatric aphakia without adequate capsular support and give a full description of their complications and visual outcomes.

\section{Transscleral Fixation with Sutures}

Over the past years, transscleral-sutured fixation of a single- or three-piece IOL has been considered an effective approach in the management of aphakia in paediatric eyes with no capsular support $[7,8]$. Nevertheless, various studies have reported significant late complications related to the sutures. These complications involve suture erosion and dislocation of the IOL 
due to suture breakage $[2,9]$. Moreover, ocular inflammation and discomfort may develop in the eyes with protruding suture ends. Close observation is required in these cases, as the risk for delayed onset endophthalmitis is increased $[10,11]$.

Asadi et al. showed a relatively high percentage of IOL dislocation resulting from suture rupture, a complication that was recorded in six of twenty-five paediatric eyes that underwent transscleral PCIOL [12]. Another study that evaluated the long-term outcomes of this surgical technique in children found that suture breakage was present approximately five years after surgery. They also observed that 10-0 polypropylene sutures had been used in all the cases complicated by suture rupture and that this rupture was mostly spontaneous [7]. Their results were supported by Price et al., who found degradation of the ruptured 10-0 polypropylene sutures after microscopic examination in patients with late dislocation of scleral-sutured IOLs [13]. It appears that long-term reliability of transscleral IOL fixation depends on the size and sturdiness of the suture material. Therefore, alternative suture size and materials such as 9-0 polypropylene or Gore-Tex have been suggested, considering the major concerns about the stability and safety of 100 polypropylene sutures used for IOL scleral fixation [7]. It is presumed that the 9-0 polypropylene suture has higher tensile strength and can better resist biodegradation and trauma [13]. Vasavada et al. observed that the 9-0 polypropylene sutures were broken clinically; however, histopathological analysis of the broken sutures was not indicative of any degenerative changes [14]. Apart from biodegradation, a clinicopathologic study concluded that polypropylene suture breakage and subsequent transscleral fixated IOL subluxation may result from the positioning IOL holes cutting the sutures and chronic inflammation that accelerates degradation process [15].

The use of alternate suture materials for IOL fixation to the paediatric sclera, including 10-0 mersilene and CV-8 Gore-Tex, has been investigated in a few studies. Although results have been encouraging, longer follow-up periods are required in order to reach safe conclusions about the stability of these materials [2].

Taking into consideration the results from published studies that investigated the use of scleral-sutured PCIOLs in children, satisfying visual outcomes have been demonstrated, with the mean postoperative best corrected visual acuity (BCVA) ranging from $0.69 \pm 0.69$ to $0.12 \pm 0.13$ logMAR at follow-up from 3 to 200 months (Table 1).

\section{Intrascleral Fixation without Sutures}

Long term side effects related to sutures led to the development of novel surgical methods for scleral IOL fixation in children. Kumar et al. presented a sutureless glued intrascleral single- or three-piece IOL fixation in 41 eyes [16]. The technique they used included externalisation of the haptics through $20 \mathrm{G}$ sclerotomies under partial thickness scleral flaps, which were then closed with fibrin glue. One case of postoperative optic capture and two cases of IOL decentration were reported. Similar results were demonstrated by Kannan and colleagues, who described a method of intrascleral IOL fixation without flaps, sutures, or glue in 40 eyes of 25 children with ectopia lentis [17]. The haptics were externalised through a scleral tunnel with the use of a $24 \mathrm{G}$ needle and buried in an adjacent scleral pocket. Four eyes developed early hyphema, five eyes developed intraocular haemorrhage, and there was one case of hypotony and one case of late IOL subluxation. Shuaib et al. compared sutureless transscleral technique for IOL fixation to retropupillary iris-claw IOLs in 30 paediatric eyes with aphakia [18]. For the IOL fixation to the sclera, they exteriorised the haptics through $23 \mathrm{G}$ sclerotomies under a scleral flap without glue usage. Postoperatively, hypotony due to subconjunctival leakage $(n=1)$, high intraocular pressure $(n=2)$, subconjunctival haptic exposure without erosion $(n=3)$, and IOL dislocation $(n=2)$ were mainly observed in the sutureless transscleral IOL fixation group. In a case series presented by Sternfeld et al., the flanged intrascleral IOL fixation (known as Yamane technique) was performed in order to correct aphakia in 12 eyes of 10 children [3]. In this technique adapted for paediatric patients, the haptics were externalised using a $30 \mathrm{G}$ thinwalled needle. Afterwards, the end of each haptic was broadened into a flange with low-temperature cautery and haptics were depressed back into the intrascleral tunnel. There was one case of postoperative IOL subluxation that also developed mild hypotony and choroidal effusion with no clinical leakage. Mild IOL decentration $(n=2)$, pigmentary deposits on the IOL $(n=3)$, irregular peaked pupil due to a vitreous strand $(n=1)$ and visible haptic through the conjunctiva in a child with Marfan syndrome were reported. Finally, a recent study retrospectively evaluated the use of Carlevale IOL in five paediatric eyes with aphakia and insufficient capsular support [19]. The Carlevale IOL is a novel foldable, acrylic, one-piece lens with T-shaped haptics, specifically designed for scleral fixation without sutures [20]. In the technique presented by Gotzaridis et al., the Carlevale IOL was inserted into the anterior chamber after three-port pars-plana vitrectomy [19]. The leading T-shaped haptic was then grabbed with $25 \mathrm{G}$ intraocular forceps under the scleral flaps and expressed through the sclerotomy. Identically, the trailing haptic was expressed through the other sclerotomy. No significant complications were reported after the surgery, and in all cases, IOL was well centered without IOL capture.

Visual outcomes of intrascleral fixated PCIOLs without sutures in paediatric population are comparable to those that have been reported for scleral-sutured IOLs. Kumar et al. found that the mean postoperative BCVA for glued intrascleral fixated PCIOLs was $0.43 \pm 0.33$ at a follow-up period of $17.5 \pm 8.5$ months (range 12-36 months) [16]. They also noted that in $53.6 \%$ of the cases, BCVA improved by more than 1 line, with no BCVA loss in any other case. Similar BCVA improvement was reported in $47.5 \%$ of the eyes that had intrascleral fixation of IOL haptics with no sutures or glue [17]. Visual results of the flanged intrascleral IOL fixation (Yamane technique) agree with those presented above, since postoperative BCVA improved in 50\% of the cases or remained stable. Significant visual acuity improvement has been reported with Carlevale IOLs as well, 
TABLE 1: The most important studies of scleral-sutured IOLs in paediatric population.

\begin{tabular}{|c|c|c|}
\hline Study & Design & $\begin{array}{c}\text { Number of } \\
\text { patients (eyes) }\end{array}$ \\
\hline
\end{tabular}

Sharpe et al. Retrospective outcomes of scleral-sutured
(1996)

Lam et al. (1998)

Retrospective safety and efficacy of scleral fixated IOLs

Key results

(1) VA improvement in six of seven patients (average improvement of 4 lines)

(2) Complications: scleral fixation suture exposure $(n=1)$, lens decentration $(n=1)$, and lens tilt $(n=1)$

(1) Good visual improvement

(2) Stable and well-positioned PCIOL after surgery in all

eyes

(3) Complications: asymptomatic pupillary IOL capture in 3 eyes

(1) Postoperative BCVA: stable in $54.5 \%$, improved by more than 1 Snellen line $27.2 \%$ and decreased by more than 1 Snellen line in $18.1 \%$

Kumar et al. Prospective case series evaluation of (1999) scleral fixated IOL implantation

$11(11)$

(2) Complications: suture erosion through the conjunctiva in $18.18 \%$, marked postoperative anterior chamber reaction in $18.18 \%$, IOL decentration in $9.09 \%$, glaucoma in $9.09 \%$, and cystoid macular edema in $9.09 \%$

(1) Postoperative BCVA: stable or improved

Zetterström et al. Retrospective long-term outcomes of (1999) scleral-sutured PCIOLs

(2) Complications: posterior synechiae $(n=4)$, cells on the

IOL surface $(n=4)$, and IOL subluxation $(n=2)$; no visual axis opacification, secondary glaucoma, or retinal complication was recorded

Vadalà et al. Retrospective results of scleral fixated

(2000)

IOLs

$3(5)$

(1) Postoperative VA: $20 / 20$ to $20 / 40$
(2) Complications: IOL dislocation $(n=1)$ and posterior capsular opacification $(n=3)$

(1) Postoperatively, BCVA within one Snellen line was achieved by more than $80 \%$ of the patients

(2) Complications: IOP increase in $11.5 \%$, marked

Jacobi et al. Prospective evaluation of transscleral (2002) fixated IOLs

$26(26)$ $19.2 \%$, and suture erosion through the conjunctiva in $7.4 \%$

Sewelam et al. Retrospective haptic position evaluation (2001) of transscleral fixated PC IOLs using UBM

Ozmen et al Retrospective assessment of the visual (2002) outcome and complications of transscleral fixated IOLs

$18(21)$

$20(20)$
IOL haptics located in the sulcus (55.0\%), anterior to the sulcus $(27.5 \%)$, and posterior to the sulcus $(17.5 \%)$

(1) Visual improvement of more than 2 Snellen lines in 9 eyes $(42.8 \%)$

(2) Complications: the most severe were concurrent endophthalmitis and retinal detachment $(n=1)$; the most common were pupillary distortion, transient pupillary membrane, pupillary capture, and strabismus and anterior uveitis

(1) Postoperative VA: improved in 70\%; in 51\% improved by two lines or more; no patient suffered visual acuity loss (2) Complications: small hyphemas (7\%), vitreous hemorrhage (5\%), ocular hypertension or hypotony (5\%) and iris capture of the IOL optic (5\%); no retinal detachment or other retinal complications were reported

(1) Postoperative VA: significantly improved $(P<0.001)$

(2) Complications: intraoperative and immediate postoperative minimal and not sight-threatening; IOL subluxation due to spontaneous 10-0 polypropylene suture breakage $(n=3)$ at $3.5,8$, and 9 years after surgery; 10 similar cases by a survey of paediatric ophthalmologists (mean, 5 years after surgery) 
TABLE 1: Continued.

\begin{tabular}{|c|c|c|}
\hline Study & Design & $\begin{array}{c}\text { Number of } \\
\text { patients (eyes) }\end{array}$ \\
\hline
\end{tabular}

(1) Postoperative BCVA: improved in $48 \%$ by $>1$ Snellen line; the main cause of reduced vision was corneal and retinal pathologies and amblyopia

Asadi and

Kheirkhah (2008)

Case series long-term results of transscleral fixated PCIOLs

Retrospective sulcus fixated, sutured

Olsen and Pribila PCIOL using endoscopic guidance during (2011) PPV
$20(21)$

$14(24)$
(2) Complications: transient intraocular hemorrhage (52\%), transient choroidal effusion (8\%), late endophthalmitis (4\%), retinal detachment (4\%), and late IOL dislocation due to breakage of polypropylene sutures after 7 to 10 years $(24 \%)$

(1) Most patients had visual function improvement

(2) Complications: suture breakage $(n=2)$ due to repeat trauma

(3) Advantages: excellent visualization and haptic localization, optimal lens centration, buried knots, broad scleral imbrication, and minimal vitreous- and hemorrhage-related complications

(4) Disadvantages: learning curve, increased operative time, long-term suture stability issues, and limited availability of intraocular endoscopes

Median postoperative BCVA: 0.2 (min: hand motion; max: 0.8$)$ in decimal notation $(P=0.017)$; BCVA improved at least one Snellen line or remained unchanged in all eyes

PCIOL: posterior chamber-intraocular lens, VA: visual acuity, BCVA: best corrected visual acuity, IOP: intraocular pressure, UBM: ultrasound biomicroscopy, and PPV: pars-plana vitrectomy.

with a mean postoperative BCVA at $0.26 \pm 0.32 \log$ MAR after a median follow-up period of 9 months (range 7-13 months) [19]. Although all these sutureless methods appear promising, they have not been widely performed in children and their long-term results need to be investigated.

\section{Iris-Sutured IOLs}

The use of iris-sutured IOLs has also been proposed for the correction of aphakia in children with no adequate capsular support. Dureau et al. described a surgical method for iris fixation of foldable IOLs in 17 eyes of 9 paediatric patients with ectopia lentis [21]. Postoperatively, they found one case of hyphema and one case of aseptic endophthalmitis; however, in all cases, IOLs were centered and pupils were round [21]. Another study presented the outcomes of iris-sutured IOL implantation in 12 eyes of children with ectopia lentis. IOL dislocation without breakage of fixation sutures was detected in four eyes (33\%). They hypothesised that these dislocations resulted from the rotation of the IOL haptic out of the suture loop [22]. Although a previous report claimed that using the remaining portion of the capsule with sufficient zonular support may be helpful in achieving better IOL stability [21], Kopel and colleagues supported that capsular remnants may be the cause of vitreous traction resulting in retinal tears and/or detachment [22]. In a similar research by Yen et al., dislocation of the iris-fixated IOL was observed in $41 \%$ of the cases [23]. Nevertheless, it was highlighted that all sutures were intact and in their appropriate iris position. Finally, researchers described the rare development of a secondary iris cyst after iris-sutured IOL insertion in two children with Marfan syndrome [24, 25].
Postoperative BCVA in paediatric cases that were corrected with iris-sutured IOLs varied from 0.24 to 0.35 $\log$ MAR at the last follow-up of 19.8 months to 4.7 years [2]. In a case series that compared the outcomes of parsplana lensectomy-vitrectomy with and without iris-sutured IOL in paediatric eyes with ectopia lentis, no statistically significant difference in the fraction of eyes that achieved BCVA of 20/40 or better was found between the two groups [22]. In addition, no difference was observed in mean postoperative BCVA. On the other hand, Shah et al. noticed that mean visual acuity improved in $71 \%$ of the cases but decreased in another $24 \%$ after iris-sutured IOL placement [26].

\section{Anterior-Fixated Iris-Claw IOLs}

Another alternative solution for the correction of paediatric aphakia in case of insufficient capsular support has been the implantation of Artisan IOL [27]. The Artisan IOL, invented by Worst in 1986, was originally a biconcave iris-enclavated IOL used in phakic patients with high myopia [28]. Due to its different design, the current biconvex rigid acrylic threepiece IOL allows aqueous flow between the optic and the iris and decreases pigment dispersion. IOL haptics are fixated to the peripheral iris, at 3 and 9 o'clock, with the use of an enclavating needle [2].

Anterior-fixated Artisan IOLs prevail over scleral- or iris-sutured IOLs because their implantation is technically easier [29]. In addition, the risk of posterior segment complications is reduced as no surgical manipulation takes place behind the iris. Nevertheless, such complications have been reported, but their occurrence is 
attributed to multiple factors, including concomitant surgical procedures such as vitrectomy or a history of previous surgery. Long axial lengths or other structural ocular deformities seem to be equally significant. For instance, axial elongation and higher lens subluxation probability appear to increase the risk of retinal detachment in patients with Marfan syndrome [2].

Anterior chamber Artisan IOLs may be complicated by pupillary block, with a cited frequency ranging from $0 \%$ to $20 \%$, and this complication can be detected from day 1 to 9 months postoperatively [2]. Hirashima et al. found that pupillary block can still be observed in pseudophakic eyes, even after laser iridotomy [29]. Surgical iridectomy was successfully performed for the inversion of pupillary block in all the reported cases. Therefore, the implantation of Artisan IOL should always involve a surgical peripheral iridectomy with sufficient size, in order to avoid this complication [2].

De-enclavation of Artisan IOLs has been recorded in up to $6.25 \%$ of cases [2]. In a study by Tychsen and Faron, repeated IOL de-enclavation led to the explantation of the Artisan IOL in one of the total 28 eyes that they were implanted [30]. Manning and colleagues reported spontaneous lens de-enclavation in one of the 16 eyes with ectopia lentis that had been treated with Artisan IOL implantation [31]. This complication occurred at the seventh postoperative year. A similar condition was described in a case series by Ong et al. [32].

The preservation of endothelial cell count (ECC) is a challenging issue for ophthalmic surgeons when performing cataract surgery and lens implantation [2]. Over the years, many studies have investigated ECL after lensectomy in children. In a prospective study by Kora et al., ECL was evaluated to be $6 \%$ three years after cataract surgery, using PCIOL and Hessberg-type anterior chamber IOLs [33]. They assumed that factors, such as age, indications for surgery, or technique, could change that result. The degree of ECL varied from $5.3 \%$ to $7.5 \%$ after extracapsular cataract surgery and PCIOL implantation in another long-term study on paediatric population [34]. Ramasubramanian and colleagues showed that mean ECC was decreased by $11 \%$ in paediatric eyes that had undergone cataract surgery [35].

Artisan IOLs concern specialists even more about ECL, because they were created for anterior chamber placement. In a number of studies that investigated the long-term results of Artisan IOLs in children with lens subluxation related to systemic disorders, the mean ECL was found to be higher, ranging from $14.2 \%$ to $18.5 \%$ [2]. Nonetheless, there are some reports that display conflicting results. Güell et al. discovered that mean ECC was not statistically different in eyes with Artisan IOL compared to eyes with any IOL-type placement [36]. Two other studies on children with lens subluxation concluded that there was no statistically significant difference in ECL between unoperated eyes and eyes that had undergone lensectomy and Artisan IOL implantation [27, 37].
It appears that prior ocular trauma may lead to even lower ECC. Odenthal and colleagues retrospectively assessed ECL after the implantation of Artisan IOL for congenital and traumatic cataract in children [38]. They observed that mean ECL was $41 \%$ in the traumatic cataract group after 10.5 years of follow-up and was strongly correlated with the original corneal scar length from trauma. Similarly, in a long-term study by Gawdat et al., a higher rate of ECC loss was noted in paediatric eyes that had undergone Artisan IOL implantation for the correction of aphakia after traumatic cataract surgery at 12-month follow-up [33]. It was assumed that original trauma and its surgical repair were liable for the greater ECL rate. However, they highlighted the necessity of longer follow-up, in order to evaluate ECL through adulthood.

Various factors are presumed to affect ECL, besides trauma. Different ECL rates have been presented among studies that explored the outcomes and complications related to Artisan IOLs and this diversity probably results from the wide variation in described surgical techniques, incisions, and follow-up durations. Considering the high heterogeneity of ECL results, researchers have not reached a safe conclusion about the correlation between ECL and the use of anterior-fixated Artisan IOLs [2].

Even though anterior Artisan IOLs have been associated with serious complications, encouraging visual outcomes have been published by multiple studies over the last two decades (Table 2). Research results showed that visual acuity was stable or improved in cases of lens subluxation that were managed with Artisan IOL placement. The mean postoperative BCVA varied from $0.36 \pm 0.26$ to $0.04 \pm 0.09 \log$ MAR at different follow-up durations [2].

\section{Retropupillary-Fixated Iris-Claw IOLs}

An alternative, retropupillary fixation of Artisan IOL has been recommended, in order to avoid ECC issues that result from anterior-fixated Artisan IOLs. Studies on the adult population have shown that, since retropupillary-fixated Artisan IOLs are located in the posterior chamber, the depth of the anterior chamber increases. Thus, the risk for ECL, which is the main concern when Artisan IOLs are used, is hypothetically lower [39, 40]. Gonnermann and colleagues presented the outcomes of retropupillary-fixated Artisan IOL use in seven paediatric eyes with lens subluxation and no capsular support [41]. A mean ECL of $6.4 \%$ was noted at the last postoperative follow-up, without spontaneous IOL dislocation or any other reported serious complication. Similar studies also revealed low complication rates with this technique $[42,43]$.

Retropupillary fixation of Artisan IOL has demonstrated good visual outcomes in aphakic children. In the study by Gonnermann et al., the mean postoperative BCVA was $0.13 \pm 0.17 \log$ MAR at a follow-up duration of $31 \pm 21$ months (range 10-64 months) [41]. These results are comparable to those reported for anterior-fixated Artisan IOLs and scleral fixated IOLs. 
TABLE 2: The most important studies of Artisan IOL in paediatric aphakic patients.

\begin{tabular}{|c|c|c|}
\hline Study & Design & $\begin{array}{c}\text { Number of } \\
\text { patients (eyes) }\end{array}$ \\
\hline
\end{tabular}

Lifshitz et al. Retrospective Artisan IOL for idiopathic (2004) subluxated lenses

$3(4)$

(1) Postoperative BCVA 6/12 or better in 3 cases that could be recorded

(2) VA improved by 2 or more Snellen lines in all eyes (3) Complications: none

(1) ECL: $41 \%$ in the traumatic cataract group

Odenthal et al. Retrospective ECL evaluation after (2006)

Artisan IOL for traumatic and congenital cataract

Sminia et al. Retrospective Artisan IOL for aphakia (2007) after trauma

(2) ECL related to the original corneal scar length of the trauma

(3) No statistical difference in ECC between operated and unoperated eye in the congenital cataract group

(1) Postoperative BCVA 20/40 or better in 4 eyes (2) Mean ECL: $40 \%$

(3) Complications: retinal detachment 19 months after primary injury in one eye

(1) Postoperative BCVA did not differ significantly between groups

Hirashima et al. Randomized controlled trial anterior vs (2010) posterior chamber iris-claw IOL lens

$16(31)$ subluxation in Marfan

(2) Complications: IOL dislocation $(n=3)$ in the PCIOL group, retinal detachment $(n=3)$ in both groups

(3) Mean postoperative foveal thickness decreased in $54.16 \%$ of the patients

Sminia et al. Retrospective ECL evaluation after (2011) Artisan IOL for traumatic and congenital cataract

Sminia et al. Retrospective Artisan IOL for ectopia (2012) lentis in Marfan

Retrospective Artisan iris-claw IOL for ectopia lentis

(2012)

$11(18)$

Siddiqui et al.

Prospective evaluation of visual outcomes

(2013) 23316948

and ECC after Artisan IOL for lens subluxation

$10(20)$

$2(4)$

Tychsen and

Faron (2013)

Prospective outcomes of Artisan IOL for aphakia

$17(28)$

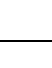

Gawdat et al (2015) Prospective outcomes of Artisan IOL for
aphakia

$18(25)$
Postoperative mean ECC comparable to the mean normal ECC for this age group reported in the literature

Good postoperative visual outcome, no serious IOLrelated complications, and ECC within the expected range for normal eyes

(1) Postoperative mean VA: $0.04 \pm 0.09 \log$ MAR $(P=0.04)$

(2) Mean postoperative ECL: $14.2 \%(P<0.001)$ (3) Complications: none

(1) Mean postoperative BCVA: $0.26 \pm 0.13 \log$ MAR $(P=0.001)$

(2) Mean postoperative ECL: $17.1 \%$

(1) Postoperatively, BCVA improved an average 2 Snellen lines (0.18 logMAR)

(2) Complications: pupillary block $(n=4)$ and deenclavation $(n=1)$

(1) Postoperative BCVA for traumatic aphakia and lens subluxation improved to $0.38 \pm 0.15 \operatorname{logMAR}(P<0.002)$ and $0.3 \pm 0.2 \log$ MAR $(P<0.0001)$, respectively

(2) One year postoperative significant decrease of ECC $\left(2892.64 \pm 441.79\right.$ cells $\left./ \mathrm{mm}^{2}\right)$

(3) Complications: traumatic dislocation $(n=2)$, pupillary block $(n=1)$

(1) Mean postoperative BCVA: $0.12 \pm 0.19 \log$ MAR

Manning et al. Retrospective outcomes after Artisan IOL

$8(16)$

(2) Mean postoperative ECL: $15.4 \%$

(3) Complications: pupillary block $(n=1)$ and deenclavation $(n=1)$

(1) BCVA improvement in both groups with no significant difference in BCVA logMAR between them

Kavitha et al. Retrospective outcomes of Artisan IOL vs. (2016) PCIOL for traumatic cataract

$50(50)$

(2) Complications in the Artisan IOL group: secondary glaucoma $(n=1)$, IOL de-enclavation $(n=1)$, and cystoid macular edema $(n=1)$

(1) Mean BCVA improved from $0.91 \pm 0.29 \operatorname{logMAR}$ to $0.18 \pm 0.23 \operatorname{logMAR}$ at final follow-up $(P<0.0001)$

Catala-Mora Prospective outcomes of Artisan IOL for et al. (2017) ectopia lentis
$12(21)$
(2) Postoperative ECL: $5.04 \% \pm 9.58 \%$ with an annual ECL rate of $3.16 \% \pm 4.46 \%$

(3) Complications: traumatic IOL dislocation and retinal detachment $(n=1)$, cystoid macular oedema $(n=1)$ 


\section{Conclusions}

Surgical correction of paediatric aphakia with inadequate capsular support represents a challenging task for ophthalmic surgeons. Several IOL designs and surgical techniques have been recommended, each one with benefits and risks. The literature has shown that scleral fixation provides good refractive and visual outcomes. Nevertheless, this method has demonstrated higher rates of complications associated with the sutures, such as suture erosion and IOL dislocation, as well as ocular inflammation and endophthalmitis. Similar complications have been described with iris-sutured IOLs, except suture breakage. Fewer cases of suture-related IOL decentration have been encountered with sutureless intrascleral IOL fixation, including the Yamane technique or the implantation of the novel Carlevale IOLs. These alternatives are potentially less complicated, but also faster. Finally, the placement of anterior Artisan IOLs is reportedly easier than that of the previous techniques; however, their long-term impact on ECL still needs to be determined.

The current literature for the correction of aphakia in children has several limitations. The majority of published reports are retrospective and noncomparative, and their sample size is small. Therefore, larger prospective studies are required in order to define which one is the optimal approach for the management of aphakia in the paediatric population.

\section{Data Availability}

No data were used to support this study.

\section{Conflicts of Interest}

The authors declare that they have no conflicts of interest.

\section{References}

[1] K. D. Epley, M. J. Shainberg, G. T. Lueder, and L. Tychsen, "Pediatric secondary lens implantation in the absence of capsular support," Journal of American Association for Pediatric Ophthalmology and Strabismus, vol. 5, no. 5, pp. 301-306, 2001.

[2] C. S. Cheung and D. K. Vanderveen, "Intraocular lens techniques in pediatric eyes with insufficient capsular support: complications and outcomes," Seminars in Ophthalmology, vol. 34, no. 4, pp. 293-302, 2019.

[3] A. Sternfeld, S. S. Taranum Basith, S. P. Kurup, and S. Basti, "Secondary intraocular lens implantation using the flanged intrascleral fixation technique in pediatric aphakia: case series and review of literature," Journal of American Association for Pediatric Ophthalmology and Strabismus, vol. 24, no. 5, pp. 286 e1-286 e6, 2020.

[4] D. A. Kumar, A. Agarwal, S. Jacob, M. Lamba, S. Packialakshmi, and A. Meduri, "Combined surgical management of capsular and iris deficiency with glued intraocular lens technique," Journal of Refractive Surgery, vol. 29, no. 5, pp. 342-347, 2013.

[5] M. Postorino, A. Meduri, L. Inferrera et al., "Scleral pockets for an innovative technique of intrascleral fixation of intraocular lens," European Journal of Ophthalmology, vol. 30, no. 5, pp. 985-990, 2020.
[6] A. Numa, J. Nakamura, M. Takashima, and K. Kani, "Longterm corneal endothelial changes after intraocular lens implantation. Anterior vs. posterior chamber lenses," Japanese Journal of Ophthalmology, vol. 37, no. 1, pp. 78-87, 1993.

[7] E. G. Buckley, "Hanging by a thread: the long-term efficacy and safety of transscleral sutured intraocular lenses in children (an American ophthalmological society thesis)," Transactions of the American Ophthalmological Society, vol. 105, pp. 294-311, 2007.

[8] M. Packer, H. I. Fine, and R. S. Hoffman, "Suture fixation of a foldable acrylic intraocular lens for ectopia lentis," Journal of Cataract and Refractive Surgery, vol. 28, no. 1, pp. 182-185, 2002.

[9] P. C. Jacobi, T. S. Dietlein, and F. K. Jacobi, "Scleral fixation of secondary foldable multifocal intraocular lens implants in children and young adults," Ophthalmology, vol. 109, no. 12, pp. 2315-2324, 2002.

[10] I. U. Scott, H. W. Flynn Jr., and W. Feuer, "Endophthalmitis after secondary intraocular lens implantation. A case-report study," Ophthalmology, vol. 102, no. 12, pp. 1925-1931, 1995.

[11] T. Heilskov, B. C. Joondeph, K. R. Olsen, and G. W. Blankenship, "Late endophthalmitis after transscleral fixation of a posterior chamber intraocular lens," Archives of Ophthalmology, vol. 107, no. 10, p. 1427, 1989.

[12] R. Asadi and A. Kheirkhah, "Long-term results of scleral fixation of posterior chamber intraocular lenses in children," Ophthalmology, vol. 115, no. 1, pp. 67-72, 2008.

[13] M. O. Price, F. W. Price Jr., L. Werner, C. Berlie, and N. Mamalis, "Late dislocation of scleral-sutured posterior chamber intraocular lenses," Journal of Cataract and Refractive Surgery, vol. 31, no. 7, pp. 1320-1326, 2005.

[14] V. Vasavada, V. A. Vasavada, R. O. Hoffman, T. S. Spencer, R. V. Kumar, and A. S. Crandall, "Intraoperative performance and postoperative outcomes of endocapsular ring implantation in pediatric eyes," Journal of Cataract and Refractive Surgery, vol. 34, no. 9, pp. 1499-1508, 2008.

[15] P. Parekh, W. R. Green, W. J. Stark, and E. K. Akpek, "Subluxation of suture-fixated posterior chamber intraocular lenses a clinicopathologic study," Ophthalmology, vol. 114, no. 2, pp. 232-237, 2007

[16] D. A. Kumar, A. Agarwal, D. Prakash, G. Prakash, S. Jacob, and A. Agarwal, "Glued intrascleral fixation of posterior chamber intraocular lens in children," American Journal of Ophthalmology, vol. 153, no. 4, pp. 594-601, 2012.

[17] K. Nb, P. Kohli, B. P. S. Pangtey, and K. Ramasamy, "Evaluation of sutureless, glueless, flapless, intrascleral fixated posterior chamber intraocular lens in children with ectopia lentis," Journal of Ophthalmology, vol. 2018, Article ID 3212740, 6 pages, 2018.

[18] A. M. Shuaib, Y. El Sayed, A. Kamal, Z. El Sanabary, and H. Elhilali, "Transscleral sutureless intraocular lens versus retropupillary iris-claw lens fixation for paediatric aphakia without capsular support: a randomized study," Acta Ophthalmologica, vol. 97, no. 6, pp. e850-e859, 2019.

[19] S. Gotzaridis, I. Georgalas, E. Papakonstantinou et al., "Scleral fixation of carlevale intraocular lens in children: a novel tool in correcting aphakia with no capsular support," Ophthalmic Surgery, Lasers and Imaging Retina, vol. 52, no. 2, pp. 94-101, 2021.

[20] I. Georgalas, D. Spyropoulos, S. Gotzaridis et al., "Scleral fixation of carlevale intraocular lens: a new tool in correcting aphakia with no capsular support," Eur Journal of Ophthalmology, Article ID 1120672121992978, 2021. 
[21] P. Dureau, P. de Laage de Meux, C. Edelson, and G. Caputo, "Iris fixation of foldable intraocular lenses for ectopia lentis in children," Journal of Cataract and Refractive Surgery, vol. 32, no. 7, pp. 1109-1114, 2006.

[22] A. C. Kopel, P. E. Carvounis, B. M. Hamill, M. P. Weikert, and E. R. Holz, "Iris-sutured intraocular lenses for ectopia lentis in children," Journal of Cataract and Refractive Surgery, vol. 34, no. 4, pp. 596-600, 2008.

[23] K. G. Yen, A. K. Reddy, M. P. Weikert, Y. Song, and M. B. Hamill, "Iris-fixated posterior chamber intraocular lenses in children," American Journal of Ophthalmology, vol. 147, no. 1, pp. 121-126, 2009.

[24] R. K. Wong and D. J. Salchow, "Iris cyst after iris-sutured intraocular lens implantation in a child," Journal of American Association for Pediatric Ophthalmology and Strabismus, vol. 16, no. 2, pp. 199-200, 2012.

[25] D. J. Salchow and J. Sinard, "Management of lens dislocation and iris cyst after iris sutured intraocular lens implantation in children with marfan syndrome," Journal of Pediatric Ophthalmology Strabismus, vol. 50, pp. e8-10, 2013, Online.

[26] R. Shah, M. P. Weikert, C. Grannis, M. B. Hamill, L. Kong, and K. G. Yen, "Long-Term outcomes of iris-sutured posterior chamber intraocular lenses in children," American Journal of Ophthalmology, vol. 161, pp. 44-49, 2016.

[27] T. Lifshitz, J. Levy, and I. Klemperer, "Artisan aphakic intraocular lens in children with subluxated crystalline lenses," Journal of Cataract and Refractive Surgery, vol. 30, no. 9, pp. 1977-1981, 2004.

[28] J. G. Worst, G. van der Veen, and L. I. Los, "Refractive surgery for high myopia. The worst-fechner biconcave iris claw lens," Documenta Ophthalmologica. Advances in Ophthalmology, vol. 75, no. 3-4, pp. 335-341, 1990.

[29] D. E. Hirashima, E. S. Soriano, R. L. Meirelles, G. N. Alberti, and W. Nosé, "Outcomes of iris-claw anterior chamber versus iris-fixated foldable intraocular lens in subluxated lens secondary to marfan syndrome," Ophthalmology, vol. 117, no. 8, pp. 1479-1485, 2010.

[30] E. M. Karasavvidou, C. Wilde, A. Zaman, G. Orr, D. Kumudhan, and G. D. Panos, "Surgical management of paediatric aphakia in the absence of sufficient capsular support," Journal of AAPOS, vol. 17, 2012.

[31] S. Manning, B. Lanigan, and M. O'Keefe, "Outcomes after lensectomy for children with marfan syndrome," Journal of American Association for Pediatric Ophthalmology and Strabismus, vol. 20, no. 3, pp. 247-251, 2016.

[32] H. S. Ong, M. Subash, and G. G. Adams, "Spontaneous subluxation of iris-claw aphakic intraocular lens causing complications in two children," J Pediatr Ophthalmol Strabismus, vol. 49, pp. e55-58, 2012, Online.

[33] G. I. Gawdat, S. G. Taher, M. M. Salama, and A. A. Ali, "Evaluation of artisan aphakic intraocular lens in cases of pediatric aphakia with insufficient capsular support," Journal of American Association for Pediatric Ophthalmology and Strabismus, vol. 19, no. 3, pp. 242-246, 2015.

[34] S. Basti, M. K. Aasuri, S. Reddy, S. Reddy, and G. N. Rao, "Prospective evaluation of corneal endothelial cell loss after pediatric cataract surgery," Journal of Cataract and Refractive Surgery, vol. 24, no. 11, pp. 1469-1473, 1998.

[35] A. Ramasubramanian, I. Mantagos, and D. K. Vanderveen, "Corneal endothelial cell characteristics after pediatric cataract surgery," Journal of Pediatric Ophthalmology \& Strabismus, vol. 50, no. 4, pp. 251-254, 2013.
[36] J. L. Güell, P. Verdaguer, G. Mateu-Figueras et al., "Unilateral iris-claw intraocular lens implantation for aphakia: a pairedeye comparison," Cornea, vol. 35, no. 10, pp. 1326-1332, 2016.

[37] M. L. Sminia, M. T. P. Odenthal, L. J. J. M. Prick, J. M. Cobben, M. P. Mourits, and H. J. Völker-Dieben, "Long-term followup after bilateral artisan aphakia intraocular lens implantation in two children with marfan syndrome," Journal of American Association for Pediatric Ophthalmology and Strabismus, vol. 16, no. 1, pp. 92-94, 2012.

[38] M. T. P. Odenthal, M. L. Sminia, L. J. J. M. Prick, N. GortzakMoorstein, and H. J. Völker-Dieben, "Long-term follow-up of the corneal endothelium after artisan lens implantation for unilateral traumatic and unilateral congenital cataract in children: two case series," Cornea, vol. 25, no. 10, pp. 1173-1177, 2006.

[39] J. J. Gicquel, S. Guigou, R. A. Bejjani, B. Briat, P. Ellies, and P. Dighiero, "Ultrasound biomicroscopy study of the verisyse aphakic intraocular lens combined with penetrating keratoplasty in pseudophakic bullous keratopathy," Journal of Cataract and Refractive Surgery, vol. 33, no. 3, pp. 455-464, 2007.

[40] M. Baykara, H. Ozcetin, S. Yilmaz, and Ö. B. Timuçin, "Posterior iris fixation of the iris-claw intraocular lens implantation through a scleral tunnel incision," American Journal of Ophthalmology, vol. 144, no. 4, pp. 586-591, 2007.

[41] J. Gonnermann, N. Torun, M. K. J. Klamann et al., "Posterior iris-claw aphakic intraocular lens implantation in children," American Journal of Ophthalmology, vol. 156, no. 2, pp. 382-386, 2013.

[42] M. Brandner, S. Thaler-Saliba, S. Plainer, B. Vidic, Y. ElShabrawi, and N. Ardjomand, "Retropupillary fixation of irisclaw intraocular lens for aphakic eyes in children," PLoS One, vol. 10, no. 6, Article ID e0126614, 2015.

[43] K. Xue and G. D. Hildebrand, "Retropupillary artisan intraocular lens implantation in very young children with aphakia following penetrating eye injuries," Journal of American Association for Pediatric Ophthalmology and Strabismus, vol. 17, no. 4, pp. 428-431, 2013. 